\title{
Nucleation Heat Treatment on the Crystalline Fraction and Crystal Morphology of Lamav Cad Lithium Disilicate
}

Bruna de Freitas Vallerini ( $\sim$ bruvallerini@hotmail.com )

Universidade Estadual Paulista Julio de Mesquita Filho Faculdade de Odontologia Campus de Araraquara https://orcid.org/0000-0002-8432-9625

\section{Mariana de Oliveira Carlos Villas-Bôas}

Universidade Federal de Sao Carlos Centro de Ciencias Exatas e de Tecnologia

\section{Lígia Antunes Pereira Pinelli}

Universidade Estadual Paulista Julio de Mesquita Filho Faculdade de Odontologia Campus de Araraquara

\section{Research Article}

Keywords: Ceramics, Thermic treatment, Crystallization, Microscopy electron scanning, X-ray diffraction

Posted Date: June 18th, 2020

DOI: https://doi.org/10.21203/rs.3.rs-34101/v2

License: (c) (i) This work is licensed under a Creative Commons Attribution 4.0 International License. Read Full License 
The authors have withdrawn this preprint from Research Square 